\title{
The Potential of Docetaxel-Loaded Poly(D,L-Lactide) Nanofibers for Preventing Recurrence after Breast Cancer Surgery in Vivo
}

\section{Qiuxia Ding ${ }^{1}$ and Shuai Shi ${ }^{2 *}$}

${ }^{1}$ Departmentof Gynaecology and Obstetrics, Xinqiao Hospital, Third Military Medical University, Chongqing, China

${ }^{2}$ Institute of Biomedical Engineering, School of Ophthalmology \& Optometry and Eye Hospital, Wenzhou Medical University, Wenzhou, China

Keywords: Docetaxel-loaded poly(d,l-lactide) nanofibers; Tumor recurrence; Drug delivery system; Breast cancer surgery

\section{Commentary}

We present here a short systematic commentary on the advantages, inadequacies and the future direction of our research work published in the 2016 study entitled "Preparation and therapeutic application of docetaxel-loaded poly (d,l-lactide) nanofibers in preventing breast cancer recurrence" [1].

The effective removal of solid tumors has always been an important aspect of cancer treatment. Unfortunately, there are often high risks of tumor recurrence after clinical operations. Electrospinning is a classical and mature method of transforming polymeric materials in the field of biomedicine and tissue engineering [2]. In our previous research, we successfully developed novel docetaxel (DTX)-loaded poly (d,l-lactide) PDLLA nanofibers, which were designed to release docetaxel in a slow and stable manner over a long period of time. Results indicated that DTX/PDLLA nanofibers exhibited apoptotic activity in 4T1 breast cancer cells with substantially reduced systemic toxicity.

It has been hypothesized that a prolonged delivery of low-dose anticancer drugs over several weeks to the resected tumor would be much more effective in preventing tumor recurrence in vivo than a delivery system of a polymer "burst" that rapidly exhausts [3].

Owing to their large surface area and their ability of site-specific delivery of drugs, electrospun fibers have been identified as a local drug delivery system. The nanofibers are also sufficiently flexible to reach the edge of tissues. Therefore, electro spun nanofibers are promising drug carriers in biomedicine, especially for postoperative local chemotherapy against cancers such as breast cancer.

A large proportion of tumor recurrences result from tumor metastasis. The DTX/ PDLLA nanofiber drug delivery system is only effective in early breast cancer patients. Because local chemotherapy is not sufficient to eradicate metastatic tumor cells, it is essential to use a combination regimen, such as multiple treatment modalities or targeted drug therapies, to treat tumor metastasis.

Thus, the local nanofiber drug delivery systems are limited to treating cancers at the early stage.

Another inadequacy of the DTX/PDLLA delivery system is their suboptimal biocompatibility and biodegradability. The nanofibers can induce a mild acute inflammatory reaction at the site of implantation. Early subcutaneous inflammation occurred with the use of DTX/ PDLLA nanofibers, along with an acidic microenvironment resulting from the degradation of PDLLA near the site of implantation.

In our experiment on tissue responses to the PDLLA nanofibers in vivo, acute inflammatory reaction was observed at week 2 , which was attenuated at week 4 without any unfavorable influences on the muscles.

Therefore, our future work should address the biocompatibility issue by optimizing the properties of the materials used to design the carriers [4] or exploring dual-biomolecule delivery systems [5,6].

In summary, our study demonstrates that DTX/PDLLA nanofibers provide a prolonged delivery of sufficient doses of cytotoxic drugs to the local tumor site, which effectively prevents local tumor recurrence following surgical resection. The DTX/PDLLA nanofibers also avoid high systemic concentrations of cytotoxic drugs, thereby minimizing systemic toxicities. However, current applications are limited to the early stage of cancer, with our future work directed towards improving the biocompatibility of the nanofiber drug delivery systems and exploring new delivery systems.

\section{Acknowledgement}

We thank Amy Tong, PhD from Liwen Bianji, Edanz Editing China (www. liwenbianji.cn/ac), for editing the English text of this manuscript.

\section{References}

1. Ding Q, Li Z, Yang Y, Guo G, Luo F, et al. (2016) Preparation and therapeutic application of docetaxel-loaded poly(d,l-lactide) nanofibers in preventing breast cancer recurrence. Drug Deliv 23: 2677-2685.

2. Yang G, Li X, He Y, Ma J, Ni G, et al. (2017) From nano to micro to macro: Electrospun hierarchically structured polymeric fibers for biomedical applications. Progress in Polymer Science.

3. Liu R, Wolinsky JB, Walpole J, Southard E, Chirieac LR, et al. (2010) Prevention of local tumor recurrence following surgery using low-dose chemotherapeutic polymer films. Ann Surg Oncol 17: 1203-1213.

4. Guo G, Fu S, Zhou L, Liang H, Fan M, et al. (2011) Preparation of curcumin loaded poly (epsilon-caprolactone)-poly(ethylene glycol)-poly(epsiloncaprolactone) nanofibers and their in vitro antitumor activity against Glioma $9 \mathrm{~L}$ cells. Nanoscale 3: 3825-3832.

5. Chang PC, Chung MC, Lei C, Chong LY, Wang CH (2012) Biocompatibility of PDGF-simvastatin double-walled PLGA (PDLLA) microspheres for dentoalveolar regeneration: a preliminary study. J Biomed Mater Res A 100: 2970-2978.

6. Haidar MK, Eroglu H (2017) Nanofibers: New Insights for Drug Delivery and Tissue Engineering. Curr Top Med Chem 17: 1564-1579.

*Corresponding author: Shuai Shi, School of Ophthalmology \& Optometry and Eye Hospital, Institute of Biomedical Engineering, Wenzhou Medical University Wenzhou, China, Tel: +86 57788833806; E-mail: shuaishi23@163.com

Received March 14, 2018; Accepted March 22, 2018; Published March 29, 2018

Citation: Ding Q, Shi S (2018) The Potential of Docetaxel-Loaded Poly(D,LLactide) Nanofibers for Preventing Recurrence after Breast Cancer Surgery in Vivo. J Oncol Transl Res 4: 130. doi: 10.4172/2476-2261.1000130

Copyright: (C) 2018 Ding Q, et al. This is an open-access article distributed under the terms of the Creative Commons Attribution License, which permits unrestricted use, distribution, and reproduction in any medium, provided the original author and source are credited. 